Linguistique, littérature, didactique

\title{
De quelques aspects du théâtre jeunesse contemporain
}

Some aspects of contemporary youth theater

\section{André Petitjean}

\section{(2) OpenEdition}

\section{Journals}

Édition électronique

URL : https://journals.openedition.org/pratiques/10477

DOI : 10.4000/pratiques. 10477

ISSN : 2425-2042

Éditeur

Centre de recherche sur les médiations (CREM)

\section{Référence électronique}

André Petitjean, « De quelques aspects du théâtre jeunesse contemporain », Pratiques [En ligne],

191-192 | 2021, mis en ligne le 15 décembre 2021, consulté le 03 janvier 2022. URL : http:// journals.openedition.org/pratiques/10477 ; DOI : https://doi.org/10.4000/pratiques.10477

Ce document a été généré automatiquement le 3 janvier 2022.

(c) Tous droits réservés 


\title{
De quelques aspects du théâtre jeunesse contemporain
}

\author{
Some aspects of contemporary youth theater
}

\author{
André Petitjean
}

\section{Introduction}

1 Parler de théâtre contemporain, c'est, un peu arbitrairement, en fonction de sa propre culture théâtrale, fixer un empan historique, (j'insisterai sur les textes produits ces vingt dernières années). C'est aussi décider d'une aire géographique ( je vais privilégier les auteurs français ou d'expression française au détriment des auteurs étrangers). C'est, enfin, chercher à dégager, au-delà de la singularité de chaque auteur, quelques principes généraux qu'un certain nombre ont en commun. Ce qui signifie que sans négliger les études à orientation philosophique et esthétique du théâtre contemporain et plus généralement de l'art dramatique, je vais traiter essentiellement, outre leurs contenus thématiques, des modes de composition de ces textes. En ce sens, mon travail se rapproche des analyses de J.-P. Sarrazac $(1981 ; 2012)$, J.-P. Ryngaert $(1993 ; 2000$; 2003), du même avec J. Sermon $(2006 ; 2012 ; 2003)$ et S. Le Pors $(2011)$ pour les personnages.

2 Alors que dans les années 1970, le «théâtre jeunesse » ou « jeune public » était quasi inexistant, les choses ont bien changé depuis. On dispose aujourd'hui, dans l'édition théâtrale, de nombreuses collections " Théâtre " proposées par différents éditeurs tels que L'École des loisirs, les Éd. Actes Sud, Éd. Théâtrales, Éd. L’Arche, Éd. Espaces 34. De fait, il existe des dizaines d'auteurs qui écrivent aussi bien pour adultes que pour les collections Jeunesse: (J.-C. Grumberg, F. Melquiot, J. Pommerat, O. Py, Durif, E. Darley...). Pour avoir une idée de la richesse et de la diversité du théâtre contemporain pour la jeunesse, on peut se reporter aux deux volumes de M. Bernanoce $(2006 ; 2012)$.

3 Si l'on en croit cette dernière, il existe, pour la seule sphère francophone, plus d'un millier de pièces et une multiplicité d'écritures théâtrales pour la jeunesse. Je n'ai donc 
pas la prétention d'en rendre compte de manière exhaustive et propose simplement quelques références de pièces que je juge particulièrement intéressantes ${ }^{1}$. Ces pièces me semblent bien adaptées au cycle 3 et certaines d'entre elles peuvent même être étudiées en seconde, dès l'instant où l'on montre que nombre de procédés de composition se retrouvent dans le théâtre dit « tout-public ».

4 Sur le plan institutionnel, la prise en compte du théâtre pour le jeune public, partout en France dans les Théâtres nationaux et dans les compagnies, et la création par Lang et Vitez, en 1973, d'un Théâtre National des enfants ont contribué à l'essor du théâtre pour la jeunesse. J'ajoute La Mousson d'hiver qui depuis 2004, en lien avec le Théâtre de La Manufacture de Nancy, fait découvrir des textes contemporains inédits de théâtre pour la Jeunesse.

5 Sur le plan institutionnel, encore, il faut citer le rôle que joue l'Éducation nationale en France dans la promotion des pièces jeunesse, en Primaire, sous la forme d'une liste officielle pour ses cycles 2 et 3, même si ces œuvres demeurent peu étudiées dans les classes, car estimées trop difficiles.

6 Du côté de certains éditeurs, l'apport pédagogique est indéniable sous la forme de «carnets» de lecture que proposent, par exemple L'école des loisirs (www.ecoledesloisirs.fr) ou Les éditions THÉÂTRALES jeunesse (www.tjeu.fr).

7 À titre d'exemple, je reprends ci-dessous la structure du carnet consacré en ligne à Autrefois, Aujourd'hui, Demain de F. Du Chaxel (2010b) ${ }^{2}$.
A. Cheminer au cœur du texte
Promenade dans le texte en abordant son titre, sa structure et l'utilisation du temps.
A. Un titre à trois temps
B. Une structure avec refrain et variations à découvrir au fil du temps de la lecture
C. Autant en emporte le temps!
B. Mise en voix/Mise en espace

8 La pièce Autrefois, aujourd'hui, demain est une partition très intéressante pour la mise en voix. Nous pourrons utilement nous reporter au travail sur le chœur et la choralité proposé pour le texte La Terre qui ne voulait plus tourner.
A. Le cercle de profération
B. La bande annonce de chaque époque
C. La prise d'espace
D. L'entrée dans l'espace
C. Mise en scène/en jeu

9 L'indication scénique de départ dans la rubrique "personnages » est une invitation à faire un travail en classe entière.
A. L'opposition collectif/intime
B. Quatre espaces en simultané sur scène
D. Annexes
A. Mise en réseau
B. Plan de travail pluridisciplinaire au CM
C. Une séquence en classe de sixième

10 Malgré cette volonté éditoriale manifeste, on peut regretter, comme l'ont relevé M. Bernanoce (2009; 2013), B. Louichon (2009), I. De Perreti (2018), le peu de place que les manuels accordent aux œuvres contemporaines. Il me semble donc utile de préciser successivement certaines caractéristiques thématiques puis les modes de composition que partagent nombre de pièces du répertoire « Jeunesse ». 


\section{Contenus thématiques}

$11 \mathrm{Au}$ niveau thématique, si l'on en juge par "l'index des thématiques " élaboré par M. Bernanoce $(2012 ; 2015 a$; 2015b), la plupart de ces pièces mettent en jeu des personnages qui figurent des enfants ou des adolescents confrontés au monde des adultes ou aux autres enfants et, plus généralement, aux vicissitudes qui affectent l'existence humaine. On y parle de leurs lieux de vie, des temps forts de leur ontogénèse, des drames de la vie (maladies, deuils, maltraitance, divorce des parents, abandon, solitude, etc.). On y traite aussi des phénomènes de société (guerres, racisme, écologie...). En ce sens, on peut dire qu'elles constituent une sorte de théâtre de "formation». J'ajoute qu'un certain nombre de pièces mettent l'accent moins sur l'enfant que sur la relation entre adultes et enfants. Ce que Marie Bernanoce (2015) appelle «le couple générationnel». Dépassant la traditionnelle figuration de l'adulte éduquant l'enfant, elles inversent les situations au sens où il revient aux enfants de dévoiler le sens de la vie, y compris aux adultes défaillants. À titre d'exemples :

Je m'appelle Non (Atlan, 1998) est l'histoire d'une jeune fille anorexique qui, devenue vieille, essaie de comprendre comment elle a fini par vaincre sa maladie.

Dans Le Jardinier (Kenny, 2006), Joe, un vieux monsieur, se souvient de son enfance, confronté à la maladie d'Alzheimer de son oncle Harry, en forme de leçon de vie.

Dans Sur la corde raide (Kenny, 2004), la petite fille est confrontée à la mort de sa grandmère et apprend à faire son deuil.

15 Les enfants de Médée (Osten \& Lydander, 2009) présente l'intérêt de faire découvrir par les enfants le mythe antique tout en abordant la problématique du divorce.

De même, Le Mioche (Aufort, 2004) met en scène un enfant enrôlé comme soldat dans un monde de bruits et de fureur.

Dans L'Adoptée (Jouanneau, 2003), un enfant tsigane se trouve confronté au préjugés racistes d'une brave paysanne avant d'être adopté par elle.

On ne manque pas non-plus de faire écho à l'actualité :

Le bruit des os qui craquent (Labeau, 2008), traite de la guerre et des enfants-soldats qui, en Afrique, sont enrôlés par l'armée ou les rebelles et livrés à la violence qu'ils subissent ou exercent. Avec La Terre qui ne voulait plus tourner, il s'agit d'aborder une réflexion de type écologique sur ce qui menace l'équilibre de la planète.

En règle générale, dans les pièces que j'ai retenues, les personnages se trouvent plutôt en nombre restreint, souvent deux, sauf dans Neige écarlate où évoluent 32 personnages. Ils sont rarement déterminés d'un point de vue sociologique. Ainsi, « un solo boy urbain » décrit Jojo dans Jojo au bord du monde de S. Jaubertie (2007) ou « Elle vit dans une famille riche, à Marseille » réfère à " Non ", dans la pièce de L. Atlan (1998) Je m'appelle Non.

Dans la majorité des cas les personnages sont plutôt indéterminés, afin de gagner en universalité et pour que les lecteurs et les spectateurs auxquels ces pièces sont destinées puissent s'identifier, quel que soit leur milieu social. Les didascalies de la liste de personnages, pauvres en informations les qualifiant - contrairement aux pièces 
classiques - le montrent bien. Soit le personnage est réduit à un patronyme, comme "Christophe », " Frobbe ", « Grobbe » dans Monsieur Fugue, soit à un statut :

•le genre : «Un jeune homme», «Une jeune fille», dans Abeilles habillez-moi de vous (Dorin, 2010) ; «Une petite fille », «Une vieille dame »;

- le métier: «Le patron du café », "La vendeuse », dans À la poursuite de l'oiseau du sommeil (Danan, J., 2010) ;

- l'activité : «Un promeneur », dans Dans ma maison de papier, j'ai des poèmes sur le feu (Dorin, 2002);

- la relation parentale : «Papa », « Maman », « Grande sœur », dans Moustique (Arca, 2011).

D'autres personnages possèdent des noms construits sur la base d'un jeu de mots: "Leboniconteur ", "Un Journanimateur" dans Le Mioche de P. Aufort (2004) ou en forme d'allégorie : «Le mangeur de Mémoire », «La Dévoreuse du temps » dans Mangemoi de N. Papin (1999). On trouve une exception inverse dans Je m'appelle Non (Atlan, 1998). Les personnages ont des noms à rallonge qui signalent un trait de caractère du type "Je parle seule mais je suis saine d'esprit ", " Rêveur écorché vif ", "Je crois aux contes de fées » ou «Le Docteur désemparé ». Il s'ensuit que les problèmes abordés dans ces pièces évitent souvent de l'être de façon réaliste mais ont plutôt recours à l'imaginaire, comme le fait S. Jaoui, dans Elles sont toutes folles (2000).

C'est pourquoi on se retrouve, dans un certain nombre de pièces, face à des récits dits "non-naturels", au sens où ce sont des fictions qui dérogent avec les lois du monde sublunaire. Il en va ainsi de la Terre qui, pour punir les hommes, décide de s'arrêter de tourner dans La Terre qui ne voulait plus tourner;. Ailleurs, les animaux parlent et interagissent avec les humains comme " Le singe » dans Les enchaînés ou " la chauvesouris » dans Là-Haut la lune (Darley, 2003). On trouve, encore, des actes de narration physiquement ou psychologiquement impossibles dans les situations de narration du monde ordinaire : «La conteuse 1 qui serait le point de vue de la grande histoire » et « «La conteuse 2, de la petite histoire» dans Petit Pierre (Lebeau, 2006 [2002]). J'ajoute les personnages sous la forme d'entités physiques anthropomorphisées: "Voix du pont », "Voix de la rivière » dans L'appel du pont (Papin, 2000). Il s'ensuit que ces récits construisent des mondes fictionnels, soit parallèles au monde réel de référence avec ses lois propres (Là-Haut, la lune; Darley, 2003), soit mêlent des éléments surnaturels à une représentation mimétique du monde réel de référence. C'est ainsi que cohabitent des personnages ordinaires et extraordinaires : «LA PETITE FILLE » et la «FÉE » dans Les Aventures d'Auren le petit serial killer; "Hippolyte, un adolescent» et "Une femmetronc » ou "Une paire de jambe d'hommes » dans Les enchaînés. Sont aussi mélangés contes et situations de télé-réalité comme dans Neige écarlate. Cela est d'autant plus patent que l'on trouve dans le répertoire «jeune public » de nombreuses réécritures contemporaines de mythes (Osten \& Lydander, 2009 ; Lebeau, 2021) et de contes (Grumberg, 2003b ; Pommerat, 2012a [2005] ; 2012b ; Py, 1999).

Il existe aussi des pièces globalement réalistes qui mettent en scène des familles dans leur quotidien, tel que Elles sont toutes folles de S. Jaoui $(2000$, p. 11) :

TABLEAU I

Salon bourgeois. Tôt le matin. C'est le début du printemps.

ÉLISE

Théo, il est presque huit heures. Il te reste cinq minutes pour avaler tes céréales.

Élise va vers la chambre, on l'entend de loin.

Théo, tu es encore en pyjama, c'est pas vrai.

Élise revient dans le salon, suivie par Théo qui trépigne. 


\section{THÉO}

Mais maman, ce n'est pas ma faute. Il y a Nina dans la salle de bains.

ÉLISE

Encore ! Elle n'est pas sortie?

\section{Les modes de composition}

Afin de comprendre ce qui est dit des personnages dans le théâtre jeunesse, il importe de décrire les modes de fonctionnement textuel de ses pièces. Pour ce faire, sans négliger les approches poétiques (Bernanoce \& Le Pors, 2018) ou didactiques (Dupuy, 2009 ; De Peretti \& Ferrier, 2016) du théâtre « jeune public ", j'ai privilégié un parti pris sémio-linguistique. Comme le rappelle, ici-même, J.-P. Sarrazac, un certain nombre d'opérations caractérisent le drame moderne (dès Ibsen, Strindberg, Maeterlinck) et surtout contemporain. On les trouve présentes dans les dramaturgies du jeune public, à savoir :

- rétrospection, avec des personnages qui racontent ou ressassent leur passé (Melquiot, 2005), pièce dans laquelle l'auteur utilise la graphie pour distinguer les personnages selon qu'ils racontent (en italique) ou rapportent leurs paroles (droit).

- anticipation à l'aide d'adresse au public sous la forme de prologues ou d'une parole chorale comme on en trouve chez E. Darley (2003) ou S. Lebeau (2008)

- répétition-variation sous la forme de reprises ou de litanies, qu'elles soient macro ou micro textuelles P. Dorin,(1997).

Il résulte de ces différents procédés un mouvement de romanisation (contamination, autant formelle que thématique, du drame par le roman) et d'épicisation (déplacement de l'action au profit de la narration) pour reprendre des catégories avancées par J.P. Sarrazac (1981).

Les textes dramatiques ont longtemps été découpés sous la forme d'actes et de scènes. Ce qui est plutôt rare dans le théâtre pour le jeunesse où l'on préfère, soit l'absence totale de découpage (Wanted Petula de F. Mequiot (2010), Une chenille dans le cour de S. Jaubertie (2008), Les deux bosssus de R. Demarcy (2008), soit des actes numérotés (1, 2, 3, 4...) comme dans En attendant le Petit Poucet (Dorin, 2001). Les scènes sont souvent courtes et multiples ( 23 pour 85 pages dans Les enchaînés; ibid., 2007). Elles sont très balisées : un titre résume la scène à venir («Le rêve de grand, Le " petit copain » de ma sœur, Ruptures, Injustice, etc. dans Arca, 2011). De même dans Salvador, l'enfant, la montagne et la mangue de S. Lebeau (1994), la pièce est découpée en 20 scènes intitulées "Souvenir ", titre complété par un résumé : "SOUVENIR 1 Le jour de ma naissance les cloches n'ont pas sonné; SOUVENIR 2 Le divin sourire de ma mère; SOUVENIR 3 Être le dixième d'une famille de huit ». Cela permet aux auteurs d'achever leur pièce par une table de matière en fin de volume. Le tout dans le but de gagner en lisibilité pour les jeunes lecteurs.

\section{Un théâtre-narration}

$\mathrm{Au}$ niveau de la composition des œuvres, il faut bien constater que les pièces contemporaines ont renoncé à la construction dramatique préconisée par Aristote : unicité d'une intrigue limitée dans son étendue, progression ordonnée et finalisée des actions, développement temporel chronologique, causalité explicite des 
enchaînements. On sait que les éléments constitutifs sont l'«ordre " (les événements s'enchaînent chronologiquement); la "complétude " (l'action forme un tout avec un début, un milieu et une fin) et l'« étendue " (la fable couvre un épisode limité de la vie du héros, dans un espace unique et une durée de 24 heures).

Toutes ces règles sont systématiquement transgressées dans bien des pièces. Pour l'« étendue » et la «complétude ", il ne s'agit plus de privilégier un moment décisif de la vie du personnage, inséré dans un temps resserré entre un début et une fin, mais de balayer une bonne partie de son existence. Ce que J.-P. Sarrazac appelle le « drame de la vie » qu' il oppose au "drame dans la vie ». Afin de compresser une vie entière dans le temps d'une représentation, ces pièces renoncent à recourir aux formes dramatiques traditionnelles (action au présent, successivité temporelle et causale, dialogues) et adoptent souvent la forme d'un théâtre-narration. Nombre de ces pièces sont fondées sur la rétrospection avec une domination de l'intrasubjectif sur les relations interpersonnelles et l'interlocution intersubjective.Soit un personnage qui parle et raconte : il assume de ce fait son double rôle de «figure narrante » et de "personnage narré ».

C'est ainsi que le personnage de "Vieille non », dans Je m'appelle Non de L. Atlan (1998, p. 11), déclare à l'ouverture de la pièce :

À la lumière du jour tombant je peins ma vie. Ceux qui l'ont traversée. Ce qui ressort quand la lumière baisse.

Comme l'indique la didascalie initiale, alternent les passages de narration où c'est tantôt « Vieille non », qui raconte ce qu'elle a vécu, tantôt « Petite non »:

«VIEILLE NON a la cinquantaine. PETITE NON : elle est la même personne, mais elle n'a que quatorze ou quinze ans »

$[\ldots]$ VIEILLE NON

À l'époque des marches, au bord du lac ou dans les près. NON mange encore. Elle mange beaucoup [...] les nourritures que sa famille s'ingénie à multiplier, et pas plus que la poésie, la nourriture ne peut la guérir des récits de celui qui deviendra son frère.

PETITE NON

Lui, par contre, il aime manger, il aime raconter, il aime rire, il aime aimer.

(ibid., p. 32).

Dans Le Jardinier de M. Kenny (2006, p. 47), Joe, le narrateur, avant de finir par un retour analeptique, « Retour au présent », revoit sa vie. Pour ce faire, l'auteur assimile le vécu du personnage au rythme des 4 saisons, chaque partie étant intitulée "Printemps", « Été », « Automne », « Hiver ».

Ce que résume Joe :

Printemps

Semer

Été

Pousser

Automne

Sorti

Hiver

Parti

De même, dans L'Ébloui de J. Jouanneau (2004, p. 7), le vieil homme qui s'adresse aux enfants pour leur raconter « l'histoire de (sa) vie » apparaît tantôt comme narrateur et tantôt comme personnage : 


\section{HORN LE VIEUX}

Le blanc de la neige, je ne pouvais le voir, pas plus que je ne pouvais voir le jardin, j'avais deux trous noirs pour seuls yeux, et tout m'était obscurité, mes enfants, même la nuit je ne la remarquais pas. Un flocon vint alors se poser dans le creux de ma main, et qui disait s'appeler « seul»

\section{HORN LE JEUNE}

Il est un peu triste ton nom, non?

SEUL

Si je le suis, oui.

\section{HORN}

Et d'où tu viens?

SEUL

Je ne le sais pas plus que toi. fille co-construisent la narration en étant tour à tour récitant (ils parlent d'eux à la troisième personne) et personnages qui dialoguent dans un rapport interlocutif.

\section{ESME}

Où est Mamie Queenie?

STAN

Te raconterai plus tard

Rentrons à la maison, c'est l'heure du goûter.

Une des poules t'a pondu un œuf.

ESME

Donc,

Papy et Esmé sont rentrés goûter à la maison.

Il n'est rien arrivé de spécial sur le chemin.

STAN

Certaine choses arrivent dont on se souvient.

\section{ESME}

Et d'autres qu'on oublie.

STAN

Ils ont parlé de choses qu'ils avaient déjà faîtes.

ESME

Et parlé de choses qu'ils n'avaient pas encore faites

Puis ils sont arrivés à la maison.

\section{STAN}

Ce n'était pas une grande maison.

Papy a fait cuire les œufs [...]

\section{ESME}

Où est Mamie?

STAN

Euh.

ESME

Viens avec moi.

Elle n'est pas dans le jardin

En train de bêcher

Ou de nourrir les poules.

Il arrive que le changement de statut (délocutif et interlocutif) se passe au sein même d'une réplique.

\section{ESME}

Dans son lit cette-nuit-là

Esmé était toujours bien silencieuse.

Papy Stan.

\section{STAN}


Qu'y a-t-il, Esmé ?

(ibid., p. 14).

Les auteurs, comme F Melquiot (2005, p. 25), peuvent user de subterfuges typographiques pour distinguer les personnages selon qu'ils racontent (italique) ou dialoguent (droit). Suzanne Lebeau utilise, elle, un caractère maigre pour « la parolerécit » et gras pour les dialogues :

MAMA BINOCLA. A quatre ans, Bouli est entré à l'École des Enfants qu'on Materne.

DADI ROTONDO. Notre nid est tombé de sa branche.

MAMA BINOCLA. A l'école, Bouli a appris des mots nouveaux.

BOULI. Gros lard. Bouffi. Baudruche. Tas de graisse.

MAMA BINOCLA. Il a pleuré les Larmes du Ventre Tordu. Il faisait trop doux dans notre nid. [...]

BOULI. Daddi, à l'école, Églantine m'a dit de me faire pilosucer.

DADI ROTONDO. Qu'elle aille se faire pilosucer elle-même, Églantine.

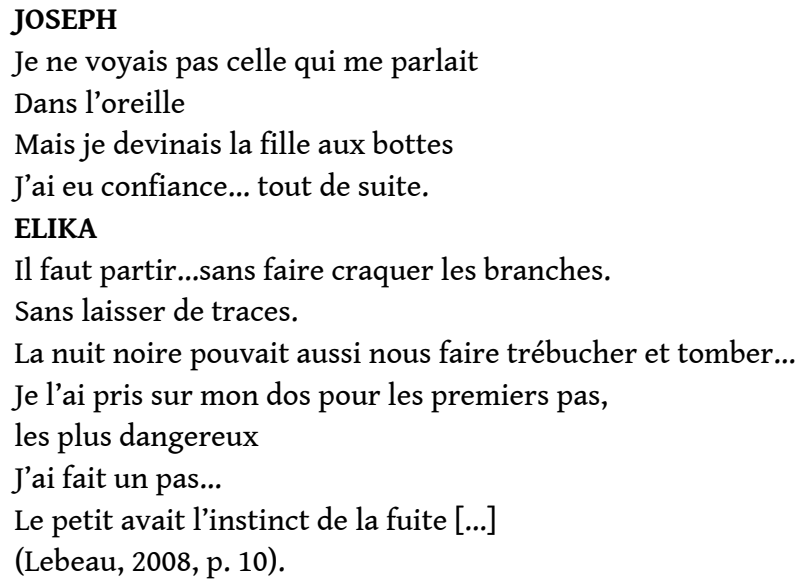

Dans tous les cas, l'effet recherché est de projeter les lecteurs dans un rapport à euxmêmes en devenir.

Autre manifestation de ce théâtre-narration, la présence d'un chœur qui s'adresse au public, soit présent au moment du prologue, soit racontant et commentant les faits tout au long de la pièce :

Images de la Terre abîmée par les hommes, images de catastrophes naturelles.

chœur.

Elle tournait, tournait, tournait

Depuis des siècles la Terre

Tournait sur elle-même

Comme une danseuse

Tournait autour du Soleil

Comme une amoureuse

Sans bruit, sans histoire

Si paisible, si polie

Si fière, si forte

Si douce, si docile

Si rassurante

Pendant ce temps

Les hommes défrichent

Creusent

Gaspillent 
Incendient

Mutilent

Se font la guerre

Puis la négligent

S'intéressent à la Lune

La trouvent

Trop grise

Trop laide

Trop froide

Reviennent sur Terre

Se font la guerre [...]

Font n'importe quoi

En font tant

Que la Terre se fâche

S'agite

Gronde

Menace

Hurle par ses tempêtes

Crache par ses volcans

Déchaîne ses océans

Puis un jour

S'arrête

Silencieuse

Immobile

(Du Chaxel, 2010a, p. 9).

\section{MENNUICESNUL}

Allésteplaît, n'hésitons plus,

Allons-nous en et rapidement,

J'entends tout près le tonnerre qui gronde,

Juste à, au-dessus de la ville

Où nous dormions hier.

\section{Le Chœur}

Ce n'est rien, ce n'est rien,

Quelques éclairs et du tonnerre,

Pour bien marquer votre départ.

C'est comme dans les films!

C'est comme dans les livres !

(Darley, 2003, p. 41)

\section{ANTIGONE}

Il a déclaré la guerre son frère,

à sa ville,

à son peuple,

à son oncle,

à ses sœurs...

La petite rivalité,

la petite jalousie entre deux frères,

est devenue une guerre sanglante entre deux armées, entre deux peuples...

\section{LE CORYPHEE}

Pourquoi les conflits des hommes, petits conflits domestiques,

banale jalousie entre deux frères,

se transforment-ils en guerres 
qui détruisent, dévastent, endeuillent?

(Lebeau, 2021, p. 36) à la stricte chronologie, avec des allers et retours fréquents entre le présent mimétique et le passé diégétique. Ce à quoi s'ajoute souvent une alternance entre narration et monstration (dialogues), si bien que l'on voyage en permanence dans le temps et l'espace.

1. Prologue derrière la fenêtre

2. Autour du hêtre dans la cour de l'école

3. Une autre fois

4. Nuit d'hiver à la fenêtre

5. L'arbre de Noël de l'école

6. Dans un cabinet de dentiste - mais est-ce bien un dentiste ?

7. Sous la fenêtre d'Auren

8. Au pied du poteau de basket,

etc.

(Danan, 2007, p. 8).

Dans Coup de bleu (Castan, 2001), la même scène se répète à l'identique plusieurs fois et l'avancée du drame est interrompue par des retours en arrière. Les deux bossus proposent deux fins différentes. Le jeu des temporalités est aussi particulièrement complexe dans Une chenille dans le cœur de S. Jaubertie (2008) dans la mesure où, dans cette fable merveilleuse, le personnage de «La présence» possède des identités plurielles - à la fois narratrice et personnage - dont l'ontologie est multiple. Si chaque changement de rôle est explicitement indiqué par les didascalies : «Et la Présence joue la mère ", "Et la Présence joue le spécialiste », "Et la présence joue les rêves », "Et la Présence joue le chêne ", "Et la Présence joue l'amour ", «Et la Présence joue la jeune femme », c'est au lecteur qu'il revient d'inférer les cadres spatio-temporels dans lesquels elle apparait.

Une autre façon de pulvériser l'ordre est de recourir à une esthétique du fragment.

\section{Une esthétique du fragment}

Nombre de ces pièces font le choix d'une composition kaléidoscopique et d'une organisation fragmentaire telle qu'elle se manifeste par des modes variés d'occupation spatiale des pages, le plus souvent à des fins expressives. P. Aufort (2004), par exemple, distribue ses pages dans Le Mioche en des blocs de taille inégale, que ce soit pour figurer la coprésence de voix simultanées ou le rendu par des onomatopées du crépitement des armes.

Autre exemple, dans certaines scènes de Le Petit violon de J.-C. Grumber (1999), le dessin se substitue aux paroles. C'est ainsi que dans la scène 3 le dessin des gants de boxe remplace iconiquement les actions des deux boxeurs en lieu et place d'un dialogue ou d'une didascalie.

Ce mode d'« épicisation » du drame peut aller jusqu'à absorber des genres de discours très divers sous la forme de blocs textuels extérieurs aux dialogues.

C'est ainsi que Jacques Rebotier, dans Les trois jours de la queue du dragon, assume cette hétérogénéité générique en incorporant dans le dialogue des genres de discours différents (lettre, publicité, exercice scolaire, proverbe...) et des type de textes variés

Pratiques, 191-192 | 2021 
(description, explication, analyse, définition). Il va plus loin encore et ajoute une ouverture intersémiotique aux images et aux partitions musicales.

Une forme textuelle est particulièrement privilégiée: la liste. Sous la forme d'inventaires d'événements, de lieux, de personnes, de métiers, d'animaux, de plantes, d'objets, les énumérations paradigmatiques ont envahi les pièces : les friandises tirées du sac dans Mange-moi de N. Papin (1999), la longue liste des essences d'arbres coupés par le bûcheron dans Une chenille dans le cour de S. Jaubertie (2008) ou celle de ce qui n'existait pas encore en «1945» dans Autrefois, Aujourd'hui, Demain de F. Du Chaxel (2010, p. 48) :

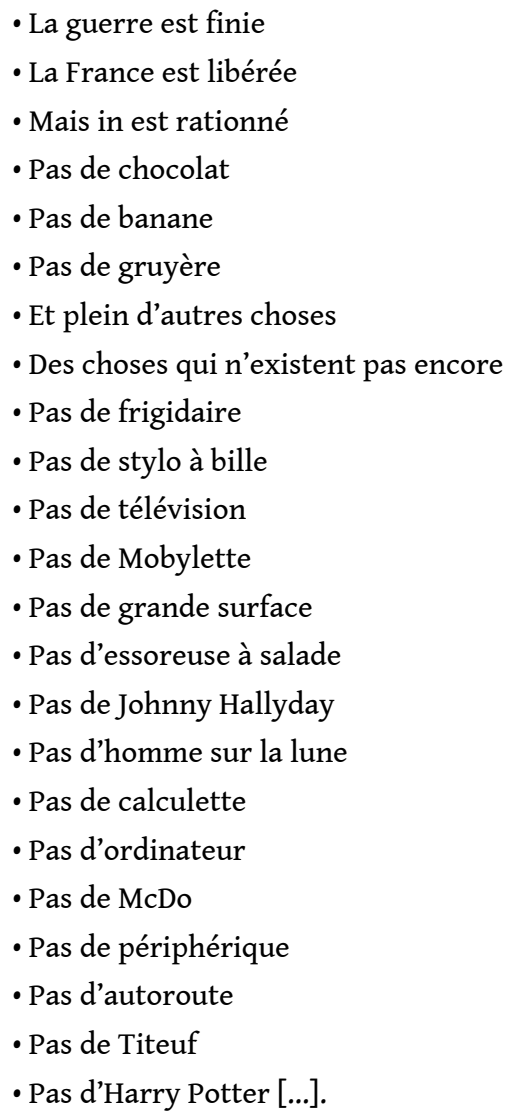

Autre aspect de la fragmentation, l'identité des personnages peut être multiple quand ils se concrétisent sous la forme de différents locuteurs qui incarnent le même personnage tel qu'il est figuré à différents âges de sa vie. Cela permet de rendre compte de leur complexité et de l'évolution de leur point de vue sur le monde. C'est ainsi que dans l'Histoire de l'oie de Michel-Marc Bouchard, le personnage de Maurice se scinde en « Maurice (Adulte) » dont la fonction essentielle est de narrer l'histoire avec son regard d'adulte et «Maurice (Enfant)» qui la vit mimétiquement mais la raconte aussi avec son point de vue d'enfant. Dans Salvador, de S. Lebeau (1994), qui s'apparente à un récit autobiographique, le personnage se dédouble sous la forme d'un narrateur adulte «Salvador » (Récit) et d'un personnage narré «Salvador » (Action) dont les paroles sont rapportées au discours direct. Dans la pièce Petite fille dans le noir, du même auteur (Lebeau, 2012), le personnage de Marie se subdivise en trois : Marie (8 ans), Marie (15 ans), Marie (35 ans). Il en va de même, enfin, dans Le Petit violon de J.-C. Grumberg (1999) qui scinde le personnage de Léo en un narrant adulte, reconnaissable par sa « perruque blanche » et un narré qui parle et agit dans un passé mimétisé. 


\section{Une écriture rythmique et poétique}

A propos de la disposition du texte, on se trouve, assez fréquemment dans ce genre de pièce, en présence d'une ponctuation blanche, au sens où il s'agit d' un mode d'occupation de l'espace (statuts des lignes, types de retraits). Son rôle est à la fois syntaxique et rythmique, comme le montrent les passages à la ligne et les sauts de ligne :

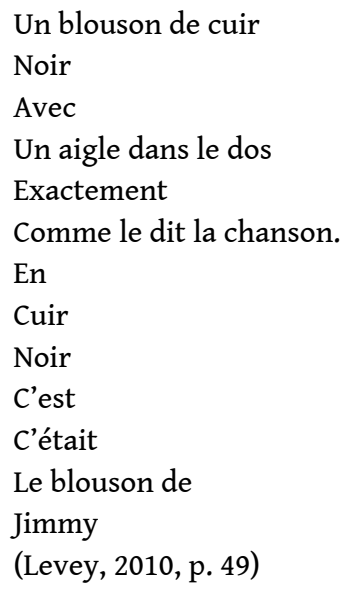

Dans certaines de ces pièces, l'écriture est composée de façon très musicale, que ce soit à l'aide de refrains ou de variations. C'est ainsi que, dans L'Appel du pont (Papin, 2000, p.13), le passage du temps et la modification entre la séparation et l'union des jeunes amants sont suggérés dans le refrain par de micro-changements (de la négation à l'affirmation) :

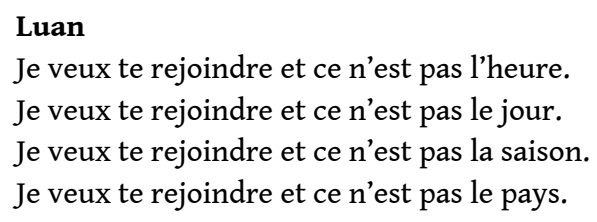

51 De même, dans Sur la corde raide (Kenny, 2004), la petite fille a grandi et arrive à faire le deuil de sa grand-mère morte (allusion au cirque), ce qu'atteste son appropriation $\mathrm{du}$ refrain : d'abord énoncé par le grand-père, il l'est à la fin par la petite fille.

\section{Le comique}

52 J'ajoute, pour finir, que ces pièces, pour sérieuses qu'elles soient, insèrent parfois des passages qui se veulent comiques, confirmant le fait qu'au sein d'une même pièce, il peut y avoir un mélange de registres. Cela se fait à l'aide de jeux de mots, de reprises intertextuelles ou de poésie ludique sur le mode d'Innocentines de René de Obaldia. 
ALIA

Qu'est-ce qu'il a?

L'OISEAU

Il est a-n-o-g-r-e-x-i-q-u-e

ALIA

Je comprends rien avec vos mots savants.

L'oiseau ramasse le dictionnaire et le donne à Alia.

ANOGREXIQUE : profond dégoût de la chair fraîche d'enfants. Les ogres peuvent en mourir....

(Papin, 1999, p. 40)

PICQ

T'as la trouille?

\section{RATON}

La courante

PICQ

Travaillera-t-on !?

\section{RATON}

Ne me donne pas d'ordre

\section{PICQ}

Je ne dis pas : «Travaille! Raton », je dis : « Travaillera-t-on !? ",

«Travaillera-t-on enfin!?» Enfin... Travaillerons-nous !?»... Enfin, travaillons, merde!

(Anne, 1997, p. 33)

LA PRINCESSE. - Tout, tout ! Je ferai tout! Tiens, je te donnerai mes bijoux, mes choux, mes poux, mes hiboux, mes cailloux, mes bijoux.

LE GRENOU. - Et tes genoux?

LA PRINCESSE. - Ah non, pas mes genoux. De l'or, si tu préfères, et mes bijoux !

(Castan, 2002b, p. 19).

\section{ENFANT 1}

Ouvrez vos livres page quatorze douze ! Vite ! Vite ! Poème : « Le lapin court dans le jardin. »

Tous répètent en chœur.

"Charlotte lui donne de la carotte. »

Tous répètent en chœur.

"Avec son frère Herbert qui mange du camembert... »

Tous répètent en chœur.

"... Tout en parlant à sa mère... »

Tous répètent en chœur.

«... Assise sur le frigidaire. »

Tous rient, répètent

"Arrive le chien... »

(Aufort, 2004, p. 35). 


\section{Le ludique}

53 On relève aussi, dans ces pièces, un goût pour la fantaisie verbale, qu'elle s'attache à dérégler le langage ou à déconstruire les expressions stéréotypées en les détournant :

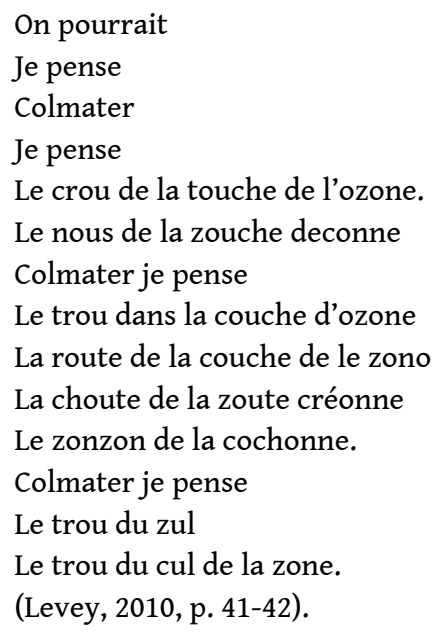

Et une vache est une vache et ce qui est fait n'est plus à faire et le temps est de l'argent et Noël au balcon Pâques où tu veux mon garçon et qui dort dîne dit le pauvre insomniaque et qui a volé un bœuf aime des omelettes et tourner sa langue sept fois pas six avant de parler au risque de qui fait le malin et qui monte dans le train et qui va à la chasse et la nuit porte conseil et porte malheur aussi il faut le dire et la nuit tous les chats et jamais deux non jamais il ne faut jamais dire fontaine je ne boirai pas parce que à chaque époque son son quoi ? Et le monde profite à ceux qui se lèvent plus tôt que plus tôt que qui et pourquoi surtout pourquoi surtout pourquoi ? Et travailler plus pour et ta sœur elle fait quoi ta sœur?

(ibid., p. 67-68).

\section{Conclusion}

55 J'espère avoir montré l'importance de ces pièces du répertoire Jeunesse pour inciter à faire découvrir, par les jeunes élèves, une pratique artistique qui ne leur est pas familière. Leur premier intérêt est de traiter de questions existentielles qui se posent au moment de l'adolescence, mais sur un mode à la fois symbolique et poétique. Leur second intérêt est d'habituer les élèves à des dramaturgies qui, transgressant les cadres génériques traditionnels, leur permettent d'apprécier dans leur différence les œuvres de facture classique. Le troisième intérêt de ces pièces, pour peu qu'on les aborde en classe, tant par le biais du jeu, de la lecture et de l'écriture, est qu'elles se prêtent particulièrement à une initiation aux pratiques scéniques. 


\section{BIBLIOGRAPHIE}

\section{Textes dramatiques}

ANNE, C. (1997). Nuit pâle au Palais. Paris : Éd. L'école des Loisirs.

ARCA, F. (2011). Moustique. Saint-Gély-du-Fesc : Éd. Espaces.

ATLAN, L. (1998). Je m'appelle Non. Paris : Éd. L'école des Loisirs.

ATLAN, L. (2004). Monsieur Fugue. Paris : Éd. L'école des Loisirs.

AUFORT, P. (2004). Le Mioche. Paris : Éd. L'école des Loisirs.

CASTAN, B. (2001). Coup de bleu. Paris : Éd. Théâtrales.

CASTAN, B. (2002a). Belle des eaux. Paris : Éd. Théâtrales.

CASTAN, B. (2002b). Neige écarlate. Paris : Éd. Théâtrales.

DANAN, J. (2010). À la poursuite de l'oiseau du sommeil. Arles : Éd. Éd. Actes Sud.

DANAN, J. (2007). Les Aventures d'Auren le petit serial killer. Arles : Éd. Actes Sud.

DANIS, D. (2006). Le Pont de pierres et la peau d'images. Paris : Éd. L'école des Loisirs.

DARLEY, E. (2003). Là-haut, la lune. Paris : Éd. L'école des Loisirs.

DEMARCY, R. (2008). Les Deux bosssus. Arles : Éd. Actes Sud.

DORIN, P. (1997). Sacré silence. Paris : Éd. L'école des Loisirs.

DORIN, P. (2001). En attendant le Petit Poucet. Paris : Éd. L'école des Loisirs.

DORIN, P. (2002). Dans ma maison de papier, j'ai des poèmes sur le feu. Paris : Éd. L'école des Loisirs.

DORIN, P. (2007). Les Enchaînés. Paris : Éd. L'école des Loisirs.

DORIN, P., (2010). Abeilles, habillez-moi de vous. Paris : Éd. L'école des Loisirs.

DORIN, P. (2013). Scur, je ne sais pas quoi frère. Paris : Éd. L'école des Loisirs.

DU CHAXEL, F. (2010a). La Terre qui ne voulait plus tourner. Paris : Éd. Théâtrales.

DU CHAXEL, F. (2010b). Autrefois, aujourd'hui, demain. Paris : Éd. Théâtrales.

GRUMBERG, J.-C. (1999). Le Petit violon. Arles : Éd. Actes Sud.

GRUMBERG, J.-C. (2003a). Marie des grenouilles. Arles : Éd. Actes Sud.

GRUMBERG, J.-C. (2003b). Le Petit chaperon uf. Arles : Éd. Actes Sud.

JAUBERTIE, S. (2007). Jojo au bord du monde. Paris : Éd. Théâtrales.

JAUBERTIE, S. (2008). Une chenille dans le cœur. Paris : Éd. Théâtrales.

JAOUI, S. (2000). Elles sont toutes folles. Paris : Éd. L'école des Loisirs.

Jouanneau, J. \& Le Pavec M.-C. (2000). Mamie ouate en Papoâsie. Arles : Éd. Actes Sud.

JOUANNEAU, J. (2003). L’Adoptée. Arles : Éd. Actes Sud. 
JOUANNEAU, J. (2004). L'Ébloui. Arles : Éd. Actes Sud.

KENNY, M. (2004). Sur la corde raide suivi de l'enfant perdue. Arles : Éd. Actes Sud.

KENNY, M. (2006). Le Jardinier. Arles : Éd. Actes Sud.

KRIBUS, S. (2010). Le Murmonde. Arles : Éd. Actes Sud.

LEBEAU, S. (1994). Salvador : la montagne, l'enfant et la mangue. Paris : Éd. Théâtrales

LEBEAU, S. (2006) [2002]. Petit Pierre. Paris : Éd. Théâtrales.

LEBEAU, S. (2008). Le Bruit des os qui craquent. Paris : Éd. Théâtrales.

LEBEAU, S. (2012). Petite fille dans le noir. Paris : Éd. Théâtrales.

LEBEAU, S. (2021). Antigone sous le soleil de midi. Paris : Éd. Théâtrales.

LESCOT D. (2014). J'ai trop peur. Arles : Éd. Actes Sud.

LEVEY, S. (2010). Cent culottes et sans papiers. Paris : Éd. Théâtrales.

MELQUioT, F. (2005). Bouli Miro, Paris : Éd. L'Arche.

MELQUIOT, F. (2010). Wanted Petula. Paris : Éd. L'Arche.

OSTEN, S. \& LYDANDER, P. (2009). Les Enfants de Médée. Paris : Éd. Théâtrales.

PAPIN, N. (1999). Mange-moi. Paris : Éd. L'école des Loisirs.

PAPIN, N. (2000). L'Appel du pont. Paris : Éd. L'école des Loisirs.

POMmerat, J. (2012a) [2005]. Cendrillon. Arles : Éd. Actes Sud.

POMMERAT, J. (2012b). Le Petit chaperon rouge. Arles : Éd. Actes Sud.

PY, O. (1999). L’Eau de la vie. Paris : Éd. L'école des Loisirs.

\section{Études}

BERNANOCE, M. (2006). À la découverte de cent et une pièces. Répertoire critique du théâtre contemporain pour la jeunesse. Paris : Éd. Théâtrales.

BERNANOCE, M. (2009). « La question du genre dans l'enseignement du théâtre contemporain ». In : Brillant-Annequin A., Bernananoce M. (coords). Enseigner le théâtre contemporain. Grenoble : SCÉRÉN-CRDP, p. 35-51.

BERNANOCE, M. (2012). Répertoire critique du théâtre contemporain pour la jeunesse. Volume 2. Paris : Éd. Théâtrales.

BERNANOCE, M. (2013). « Le répertoire théâtral dans son contexte scolaire, à l'épreuve des genres et des esthétiques », Le Français Aujourd'hui 180, p. 27-38.

BERNANOCE, M. (2015a). « Le couple générationnel dans le théâtre jeunesse : une dualité peut en cacher une autre ", Recherches \& Travaux 86, p. 83-92.

BERNANOCE, M. (2015b). « Le Répertoire de théâtre jeunesse : des esthétiques contagieuses », Recherches et Travaux 87, p. 23-38.

BERNANOCE, M. \& LE PORS, S. (2018). Poétiques du théâtre Jeunesse. Arras : Artois presses université. 
DE PERETTI, I. (2018). Théâtre à l'école et didactique de la littérature : contradictions, apports, tensions (1970-2017). Habilitation à diriger des recherches : Université d'Artois.

DE PERETTI, I. \& FERRIER, B. (2016). Théâtre d'enfance et de jeunesse, de l'hybridité à l'hybridation. Arras : Artois presses université.

DUPUY, C. (2009). « Transmettre le théâtre pour la jeunesse en formation initiale ». In : BrillantAnnequin, A. \& Bernanoce, M. (dirs). Enseigner le théâtre contemporain. Grenoble : SCÉRÉN-CRDP, p. $145-154$

LE PORS, S. (2011). Le Théâtre des voix. À l'écoute du personnage et des écritures contemporaines. Rennes : Presses universitaires de Rennes.

Louichon, B. (2009). « Le Théâtre au cycle 3 ». In : Brillant-Annequin, A. \& Bernanoce, M. (dirs). Enseigner le théâtre contemporain. Grenoble : SCÉRÉN-CRDP, p. 133-144.

RYNGAERT, J.-P. (1993). Lire le théâtre contemporain. Paris : Dunod.

RYNGAERT, J.-P. (2000). « Ecritures contemporaines : la disparition des repères ", Dix-neuf/vingt. Revue de littérature moderne 9, p. 87-99.

RYNGAERT, J.-P. (2003). « Écritures théâtrales contemporaines : état des lieux », Pratiques 119-120, p. 109-118. En ligne : https://www.persee.fr/doc/prati_0338-2389_2003_num_119_1_2016.

RYNGAERT, J.-P. \& SERMON, J. (2006). Le Personnage théâtral contemporain :décomposition, recomposition. Paris : Éd. Théâtrales.

SARRAZAC, J.-P. (1981). L'Avenir du drame. Lausanne : Éd. L'aire.

SARRAZAC, J.-P. (2012). Poétique du drame moderne. Paris : Éd Le Seuil.

SERMON, J. (2003). « Le personnage contemporain et ses états... (de paroles) », Pratiques 119-120, p. 119-130. En ligne : https://www.persee.fr/doc/prati_0338-2389_2003_num_119_1_2017.

\section{NOTES}

1. Voir la partie « Textes dramatiques » de la bibliographie.

2. Accès : https://www.editionstheatrales.fr/pedagogique/les-carnets/la-terre-qui-nevoulait-plus-tourner-autrefois-aujourd-hui-demain/autrefois-aujourd-hui-demain/ cheminer-a-l-interieur-du-texte

\section{RÉSUMÉS}

Le but de cet article est d'explorer le théâtre pour la jeunesse contemporain en me focalisant plus particulièrement sur quelques caractéristiques des personnages dans ce répertoire. Au niveau thématique, la plupart de ces pièces mettent en jeu des personnages qui figurent des enfants ou des adolescents confrontés au monde des adultes ou aux autres enfants et, plus généralement, aux vicissitudes qui affectent l'existence humaine. En ce sens, on peut dire qu'elles constituent 
une sorte de théâtre de « formation ». Il reste qu'elles évitent le piège du didactisme en recourant à un mode de composition kaléidoscopique et d'une organisation fragmentaire qui dérogent aux règles classiques. Un autre intérêt de ces pièces est que la plupart gagnent à être accompagnées d'un travail de mise en scène à mener avec les élèves.

The aim of this article is to explore contemporary youth theater by focusing more particularly on some characteristics of the characters in this repertoire. Thematically, most of these plays involve characters who represent children or adolescents confronted with the world of adults or other children and, more generally, with the vicissitudes that affect human existence. In this sense, we can say that they constitute a kind of "training" theater. The fact remains that they avoid the trap of didacticism by resorting to a mode of kaleidoscopic composition and a fragmentary organization which derogates from the classical rules. Another advantage of these plays is that most benefit from being accompanied by staging work to be carried out with the students.

\section{INDEX}

Mots-clés : Théâtre de la jeunesse, répertoire contemporain, contenus thématiques, modes de composition

Keywords : Youth theater, contemporary repertoire, thematic contents, modes of composition

\section{AUTEUR}

\section{ANDRÉ PETITJEAN}

Université de Lorraine, Crem, F-57000 Metz, France 